\title{
Androgenetische Alopezie
}

Dr. med. Pierre A. de Viragh

Universitätsklinik für Dermatologie, Inselspital, Universitätsspital Bern, und Privatpraxis, Zürich

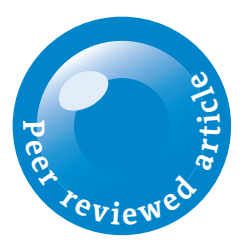

Die Diagnose einer androgenetischen Alopezie ist einfach, weil immer zutreffend, handelt es sich ja um einen physiologischen Alterungsprozess. Nur bei einer für das Alter übermässigen Ausprägung ist eine Therapie notwendig. Wichtig ist dann das Erkennen von Simulatoren der AGA, welche die Haarausdünnung über das genetisch Vorgegebene hinaus beschleunigen, oder eine konstitutionelle AGA imitieren, und nach ganz anderen Therapieansätzen verlangen.

\section{Einführung}

Die androgenetische Alopezie (AGA) ist mechanistisch ein Telogeneffluvium, mit - bedingt durch unterschiedliche Androgensensitivität der Kopfhautregionen - pathognomischer elektiver Verteilung am Kopf; bei Mann und Frau ist die AGA zwar etwas unterschiedlich ausgeprägt, aber bei beiden ist das Occipitum ausgespart («zentrale» oder «frontale» Verteilung, engl. "patterned" alopecia) (Abb. 1A). Dies ist im Gegensatz zum diffusen Telogeneffluvium, welches die Gesamtheit der Haarfollikel betrifft (Abb. 1B). Die genaue Wirkweise der Androgene nach dem Eintritt in die Haarfollikelzellen ist unbekannt, sei es, dass sie in normaler Menge vorliegen und entsprechend der genetischen Empfindlichkeit ihre Wirkung am Haarfollikel entfalten, oder sei es bei absoluter oder relativer Hyperandrogenie. Dabei verhält sich der Effekt am Kopf gegenläufig zur Wirkung am Körper (Glatze versus Bart usw.). Bei Polymorphismen zahlreicher beteiligter Gene und deswegen individueller Unterschiede (Affinität und Aktivität der Androgenrezeptoren, Aktivität der 5a-Reduktase, Aromatase, Lipoxygenase, Steroid-Sulfatase usw.) lösen sie am Kopf, mit jedem Haarzyklus zunehmend, eine charakteristische Miniaturisierung der Follikel aus: Verminderung des Durchmessers der betroffenen Haare und so in der Lupenuntersuchung unterschiedliche Haarkaliber ("Anisotrichose») sowie Verkürzung ihrer Wachstumsphase (Anagen) mit frühem Übertritt in die Ruhephase (Telogen) und dadurch Verkürzung der Haarlänge.

Als physiologischer Prozess kann die AGA selten als erstes Zeichen der Pubertät erscheinen (präpubertäre AGA) oder während dieser auftreten (adoleszente AGA). Spätestens ab Ende der Pubertät liegt sie bei allen in diskreter Form vor und akzentuiert sich sichtbar über die Jahre. Die Diagnose einer AGA ohne Angabe der Ausprägung und ohne Abgleichung mit dem für das Alter Nor- male ist deswegen sinnlos (gängige Einteilungen: Hamilton-Norwood für den männlichen, Ludwig für den weiblichen Typ). Bei über der Norm liegender Ausprägung kann die AGA behandelt werden, nicht ohne bei Vorliegen von Hinweisen für eine Hormonstörung (schnelle Verschlechterung, Hirsutismus, Zyklusstörungen) weitergehende endokrinologische Untersuchungen zu veranlassen (Tab. 1) [1]. Simulatoren der AGA (Tab. 2) können diese falsch als übermässig erscheinen lassen, was zu therapeutischen Fehlschlägen, und daraus zu therapeutischem Defätismus oder zu unnötigen Untersuchungen führt. Hierbei sind immer auch die individuellen Unterschiede im Reaktionsmuster auf die Androgene und die Kofaktoren des Haarwachstums aufgrund genetischer Unterschiede anzuerkennen - nicht alle werden bei gleichen Umständen mit einem Haarausfall reagieren, und nicht alle werden auf die gleiche Therapie gleich ansprechen [2].

\section{Simulatoren der AGA}

\section{Diffuses Telogeneffluvium}

Um eine Haarausdünnung subjektiv überhaupt zu bemerken, muss diese objektiv bereits bedeutsam sein (je nach Schätzung 20-50\% der Haare). Eine übermässige AGA kann fälschlich angenommen werden, wenn die bisher inapparente in normalem Ausmass altersgerecht ablaufende Haarausdünnung, durch ein diffuses Telogeneffluvium akzentuiert wird, mit inspektorisch noch normal scheinender Haarmenge okzipital. Das Telogeneffluvium kann akut oder subakut ablaufen und führt dann mehr oder weniger schnell zur globalen Haarverminderung. Wenn ein Trichogramm zur Objektivierung der Haarausfallsquote nicht möglich ist, muss anamnestisch eine schleichende von einer schnellen Verschlechterung der Haare unterschieden 

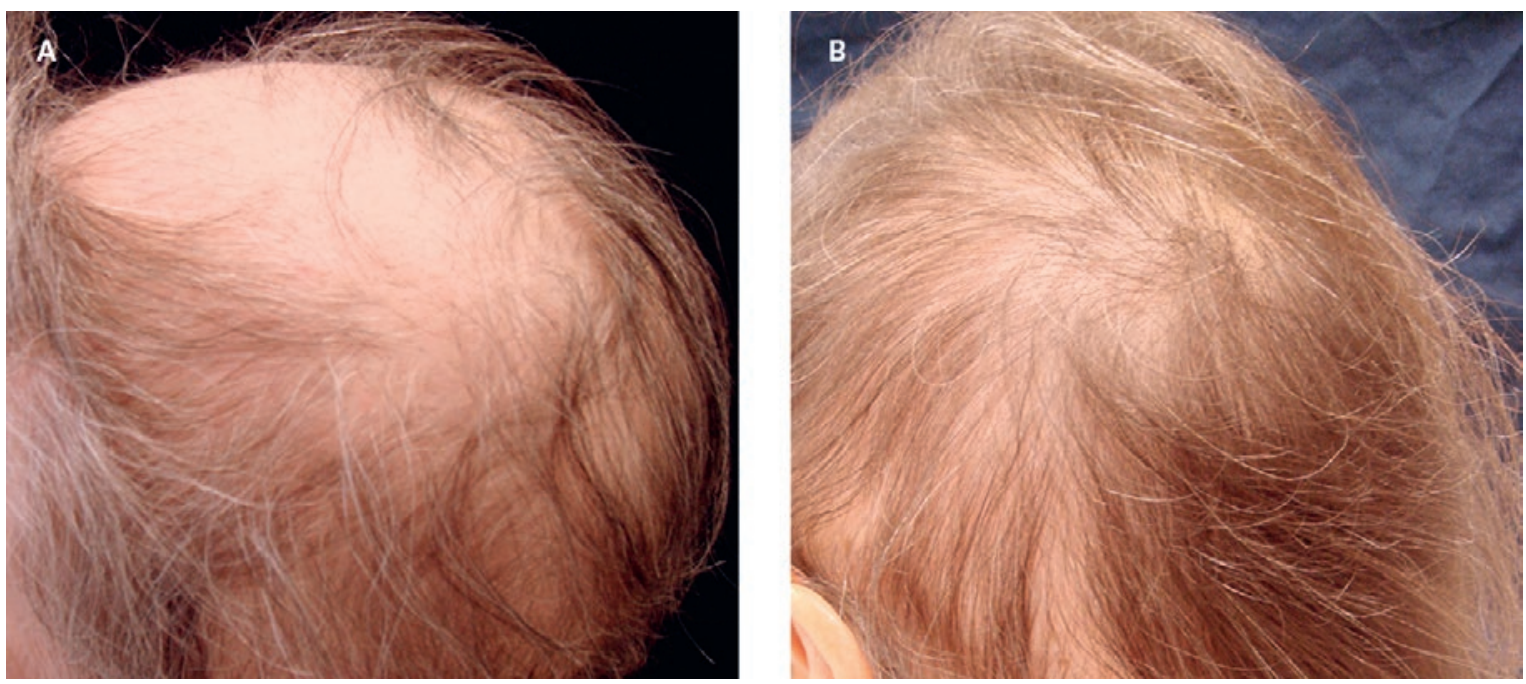

Abbildung 1: A: Fortgeschrittene AGA, Haarausfall unter Aussparung des Occipitums. B: Diffuses Telogeneffluvium, Haarausfall mit Einbezug des Occipitum.

Tabelle 1: Laboruntersuchungen bei Haarausfall. Hormonuntersuchungen sind kostspielig und bei regelmässigem Zyklus selten indiziert.

Systematisch zu untersuchen

ANA, TSH, Ferritin inkl. CRP und ALAT (um Ferritinerhöhungen bei Hepatopathien oder Entzündungen auszuschliessen), Zink, aktives Vitamin $B_{12}$ (Holotranscobalamin), Biotin. Im Kontext: Syphilis-/HIVScreening.

+ bei Adipositas (nüchtern) SHBG. HOMA-Index oder Insulinresistenz.

+ bei Verdacht auf Endokrinopathie, va bei Zyklusunre-

- Ältere Patientin bei Tumorverdacht: Testosteron total, DHEAS (von Zyklus unabhängig).

gelmässigkeit

Blutentnahme in der follikulären Zyklusphase, vorzugsweise 3.-5. Tag, morgens, nüchtern, ggf. nach einer Pil-

- Junge Patientin: zusätzlich LH/FSH, $\mathrm{E}_{2}$, Androstendion, 17-OHlenpause von mindestens zwei Spontanzyklen.

Progesteron, Prolaktin. Ggf. endovaginaler Ultraschall der Ovarien zum Ausschluss von PCO (häufigste Ursache).

+ bei Verdacht auf hypoöstrogenes Effluvium durch KOK $E_{2}$ (hier keine Pillenpause! Blutentnahme jederzeit, idealerweise nach der Menstruationspause, vor Beginn des nächsten Pillenzyklus).

ALAT = Alanin-Aminotransferase; ANA = antinukleärer Antikörper; CRP = C-reaktives Protein; DHEAS = Dehydroepiandrosteronsulfat; $E_{2}=$ Östradiol; FSH = Follikel-stimulierendes Hormon; $\mathrm{KOK}=$ kombinierte Kontrazeption; $\mathrm{LH}=$ luteinisierendes Hormon; $\mathrm{PCO}=$ polizystische Ovarien; SHBG = Sexualhormon-bindendes Globulin.

Tabelle 2: Simulatoren der androgenetischen Alopezie.

\begin{tabular}{|c|c|}
\hline \multirow[t]{2}{*}{ Telogeneffluvium, diffus } & Subakut \\
\hline & Akut \\
\hline \multirow[t]{3}{*}{ Hormonelle Effluvien } & Pseudohormonelle Alopezie \\
\hline & Hypoöstrogene Alopezie \\
\hline & latrogene androgenetische Alopezie \\
\hline \multirow[t]{2}{*}{ Anageneffluvium } & Chemotherapie-induzierte Alopezie, permanent \\
\hline & Alopecia areata incognita \\
\hline \multirow[t]{3}{*}{ Vernarbende Alopezien } & $\begin{array}{l}\text { Fibrosierende Alopezie } \\
\text { - frontal (M. Kossard) } \\
\text { - zentral (M. Trüeb) }\end{array}$ \\
\hline & Traktionsalopezie \\
\hline & Druckalopezie \\
\hline \multirow{4}{*}{$\begin{array}{l}\text { Konstitutionelle } \\
\text { Effluvien/Alopezien }\end{array}$} & Kongenitale temporale trianguläre Alopezie \\
\hline & Chronisches Telogeneffluvium \\
\hline & Psychopathologien \\
\hline & Genetische Syndrome \\
\hline
\end{tabular}

werden. Bei schneller Progression werden mögliche Ursachen für ein Telogeneffluvium durch die angezeigten Untersuchungen ausgeschlossen (Tab. 1 und 3). Bei Durchführen eines Trichogramms ist die korrekte Interpretation wesentlich. Der normale Haarausfall (Haarerneuerung im Mosaik) erfolgt wellenartig mit Phasen verstärkten und verminderten Ausfalls; dessen «Amplitude» schwankt zwischen 15 und 20\% Telogenhaaren. Eine Verminderung unter 15\% ist gleichbedeutend mit einer Verstärkung über 20\%. Einer kahl werdenden Patientin «gutes Haar» zu bescheiden, nur weil ihr Telogenwert z.B. bei 5\% liegt, ist eine grobe Falscheinschätzung. Diese Fehlinterpretation kann sowohl bei der AGA wie auch dem diffusen Effluvium passieren.

Häufige Ursache für einen globalen Haarausfall ist der Mangel an einem Mikronährstoff. Wichtigster Mangel ist hierbei der Eisenmangel auch ohne Anämie; er ist 
Tabelle 3: Differentialdiagnose des subakuten oder akuten Telogeneffluviums. Dieses tritt 2-3 Monate nach einem das Haarwachstum schwächer oder heftiger kompromittierenden Ereignis in Erscheinung, wo die Haarfollikel frühzeitig in die Ruhephase Telogen getrieben werden. Beim Anageneffluvium erfolgt ein eigentlicher Haarausfall innert 2-3 Wochen nach einer direkten Schädigung des Haarfollikels, z.B. nach akuter Vergiftung, Chemotherapie oder bei Alopecia areata.

Post partum und Absetzen der «Pille», nach Abstillen

Hypo- und Hyperthyreose (CAVE: Dysthyroïdie postpartal)

Postinfektiös / Fieber $>39^{\circ} \mathrm{C}$ oder chronischer Infekt (inkl. HIV, Lues)

Psychische und physische Traumata, inkl. chirurgische Eingriffe

Kollagenosen (bei 7\% Erstsymptom von systemischem Lupus)

Aggressive Reduktionsdiät und Kachexie/Anorexie

Malnutrition, inkl. Vegetarismus, Magenbanding, Koffeinexzess usw., parenterale

Ernährung

Chronische Krankheiten (inkl. Krebserkrankung, ggf. noch unerkannt)

Leichte Vergiftungen

Medikamente durch Nichtverordnen einer Erhaltungstherapie; schliesslich bleiben die Umstände, welche zum Haarausfall/Eisenmangel geführt haben, bestehen (Vegetarismus, Menorrhoe, Absorptionsstörung). Die Indikation zum Abklären eines Eisenmangels richtet sich nach den Regeln der inneren Medizin. Erinnert sei an das Haar als träges System: eine Verbesserung erkennt man erst nach 9-12 Monaten.

\section{Pseudohormonelle Alopezie}

Einzelne Patienten zeigen eine nur zentral mässig verstärkte Haarausdünnung mit schleichendem Verlauf, charakteristisch für eine klassische AGA; auch im Trichogramm ist objektiv der Haarausfall nur zentral aktiv. Im Labor zeigt sich ein Eisenmangel und/oder ein Vitamin- $\mathrm{B}_{12}$-Mangel. Hier kann die alleinige Korrektur des Mangels die AGA umkehren. Diesen «pseudohormonellen Haarausfall» kann man bei Eisenmangel mit der eingeschränkten Funktion zweier haarrelevanter Enzyme im Androgenmetabolismus erklären: Verminderte Aktivität der eisenabhängigen Aromatase zur Synthese von Östrogen und verminderte Hemmung der 5a-Reduktase durch Metaboliten der eisenabhängigen Lipoxygenase. Beide führen im Teufelskreis zu einer lokalen relativen Hyperandrogenie und Hypoöstrogenie (Abb. 2). So kommt es bei einem (prä-)latenten Eisenmangel zu einer verstärkten AGA [4]. Andererseits erklärt sich die Aktivierung einer AGA bei Vitamin- $B_{12}$-Mangel durch die dann verminderte Methylierung und Inaktivierung der Androgenrezeptoren, mit dadurch verstärkter Androgenwirkung. zum Haarausfall bei Patienten unterschiedlich sind [3], eine Annahme, welche in Zeiten individualisierter Medizin nicht mehr anstössig sein dürfte. Zusätzliche Symptome eines möglichen Eisenmangels (Nagelbrüchigkeit, Fatigue, Stimmungsschwankungen usw.) begründen die Entscheidung zur Behandlung mehr als der einfache Ferritinwert; dieser ist sowieso - daran sei wieder einmal erinnert - kein absoluter Wert, sondern ein Schätz- und Wahrscheinlichkeitswert, im Knochenmarksausstrich einen Eisenmangel vorzufinden. Die Interpretation muss also im klinischen Kontext erfolgen. Bei Haarausfall ist ein Ferritin von mindestens 50-70 $\mu \mathrm{g} / \mathrm{l}$ empfohlen. Die individuelle Schwelle schwankt erfahrungsgemäss im Patienten selbst, wo sie gegebenenfalls durch weitere Mangelzustände zusätzlich gesenkt wird. Häufige Ursachen, wenn keine Verbesserung des Haarwuchses durch Eisen zu erreichen ist, sind: (1.) Ungenügendes Anheben der Eisenreserven (im besten Fall Anstieg des Ferritins um 0,25 pro Eisentablette, um 10 pro 100 mg i.v.-Eisen; Kontrolle 3 Wochen nach der letzten Tablette, 8 Wochen nach der Infusion), (2.) erneutes Abfallen des Eisens

\section{Hypoöstrogene Alopezie}

Östrogen hat eine das Haarwachstum direkt stimulierende Wirkung, was auch in der Zellkultur nachgewiesen wird. Es erklärt das schöne Haar während der Schwangerschaft und den Haarausfall im Östrogentief nach der Niederkunft. Auch stimuliert das Östrogen die Testosteron-abbauende Aromatase. So kann jeder Abfall des Östrogens bei Frauen zu einem diffusen, aber auch zu einem AGA-ähnlichen Haarausfall führen: Menopause, Therapie mit Antiöstrogenen und Antiaromatasen, aber auch eine hormonelle Antikonzeption mit einem einfachen Gestagen-Präparat nadotropine $\mathrm{zu}$ einer relativen Hypoöstrogenie. So wirkt sich bei zur AGA-neigenden Patientinnen eine Kontrazeption mit einer Minipillle während der Stillzeit, im postpartalen Östrogentief, ganz verheerend aus. Auch der paradoxe verstärkte Haarausfall bei Einzelnen selbst unter antiandrogener kombinierter Kontrazeption (KOK) erklärt sich hieraus, wo vor allem das (Minipille). Diese führen durch die Hemmung der Go- 


\section{Testosteron}

Gammalinolensäure (GLA)

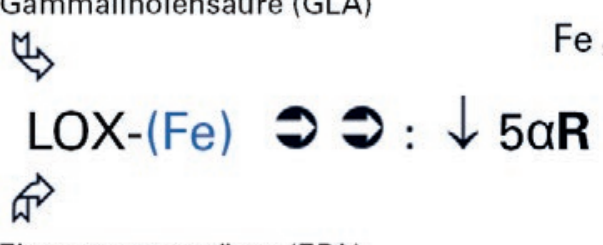

Eicosapentaensäure (EPA)

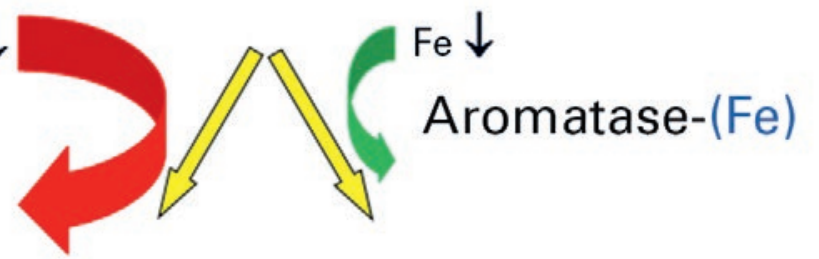

DHT Östradiol

Abbildung 2: Mechanismus der pseudohormonellen Alopezie (Annahme). Testosteron wird einerseits durch die eisenabhängige Aromatase zu Östradiol (proanagen, reguliert weiter die Aromatase auf) und andererseits durch die 5a-Reduktase (5aR) zum potenterem Dihydrotestosteron (DHT) umgewandelt; die 5 aR-Aktivität wird durch Metaboliten des Fettsäurestoffwechsels gehemmt, welche von der eisenabhängigen Lipoxygenase (LOX) abhängen. Bei Gewebeeisenmangel ist die Aromatase weniger aktiv, mit Rückstau an Testosteron. Dieses wird dafür umso effizienter durch die ungehemmte $5 \mathrm{aR}$ zu DHT umgewandelt, wegen verminderter Aktivität der LOX.

Gestagen die Gonadotropinhemmung bewirkt und das exogene Ethinylestradiol das endogene Östrogen $\left(E_{2}\right)$ nicht in allen Gewebekompartimenten ersetzen kann. Bei diesen Patientinnen, besonders wenn das $E_{2}$ mit der KOK deutlich unter den tiefsten Wert im ovulatorischen Zyklus abgesenkt wird, muss nicht Cyproteronacetat zur KOK zugesetzt, sondern diese ganz abgesetzt und durch eine nicht-hormonelle Kontrazeption ersetzt werden.

\section{Iatrogene androgenetische Alopezie}

Eine KOK oder ein Hormonersatz mit einem Gestagen erster oder zweiter Generation kann wegen deren androgenisierenden Teilwirkung zu einer iatrogenen AGA führen; hierzu gehören auch dosisabhängig die Hormonspiralen und Tibolon im Hormonersatz. Bei aktuell aktiver Organisation im Internet von Geschädigtengruppen ist vor Verordnung einer Hormonspirale die Veranlagung zur AGA auszuschliessen und auf die mögliche Nebenwirkung hinzuweisen. Die Therapie besteht - bei Fehlen von Kontraindikationen - im Wechsel der «Pille» oder des Hormonersatzes zu einem Kombinationspräparat mit einem Gestagen neutraler (dritte Generation) oder antiandrogener Wirkung (vierte Generation: Cyproteronacetat, Drospirenon, Chlormadinon, Dienogest, Nomegestrol).

Permanente Chemotherapie-induzierte Alopezie Bei der Chemotherapie sind die Umstände analog der Demaskierung einer AGA durch ein Telogeneffluvium. Auch eine Chemotherapie-induzierte Alopezie, ein Anageneffluvium, kann - wenn sie nicht total ist - eine physiologische «zentrale» Haarausdünnung akzentuieren. Dies ist im allgemeinen reversibel und im unmittelbaren Zusammenhang mit der Krebsbehand- lung auch offensichtlich. Weniger bewusst ist der Umstand, dass die neuen Chemotherapie-Schemata (Therapeutika, Kombinationen, Wiederholungen) auch zu einem totalen oder partiellen Haarverlust führen können, welcher permanent ist, mit definitiver Inaktivität der Follikel; so wird eine übermässige AGA imitiert, insbesondere, wenn sie im Verlauf durch Antiaromatasen oder -östrogene weiter verstärkt wird.

\section{Alopecia areata incognita, systemischer Lupus erythematodes, Syphilis}

Die Alopecia areata, auch diese ein Anageneffluvium, führt zu völlig kahlen, umschriebenen Stellen, oder zur vollständigen Glatze. Eine wenig bekannte Sonderform ist die Alopecia areata incognita, welche eben nicht erkannt wird. Sie tritt schleichend auf und äussert sich als einziger Hinweis durch eine etwas unregelmässige diffuse Haarausdünnung ohne Kahlstellen; sie imitiert wie das Telogeneffluvium - durch Demaskierung eine fortgeschrittene AGA, oder sie imitiert ein akutes diffuses Telogeneffluvium, für welches dann keine Ursache gefunden wird. Die klassischen «Ausrufezeichenhaare» der Alopecia areata fehlen meist. Auch in der Histologie fehlt das klassische "Bienenschwarm-artige» peribulbäre Infiltrat, so dass es einiger Erfahrung und vor allem einer genügend grossen Biopsie bedarf, um die Diagnose zu stellen (Abb. 3). Selbstredend ist dann die Therapie als AGA nutzlos: ein völliges Versagen der AGA-Therapie sollte die Erstdiagnose anzweifeln lassen, ebenso wie Versagen der Behebung vermuteter Mangelzustände. Therapie ist die systemische Immunsuppression (Kortisolpulse, Methotrexat). Ähnlich können sich der Haarausfall im Rahmen eines systemischen Lupus und einer Lues darstellen. 

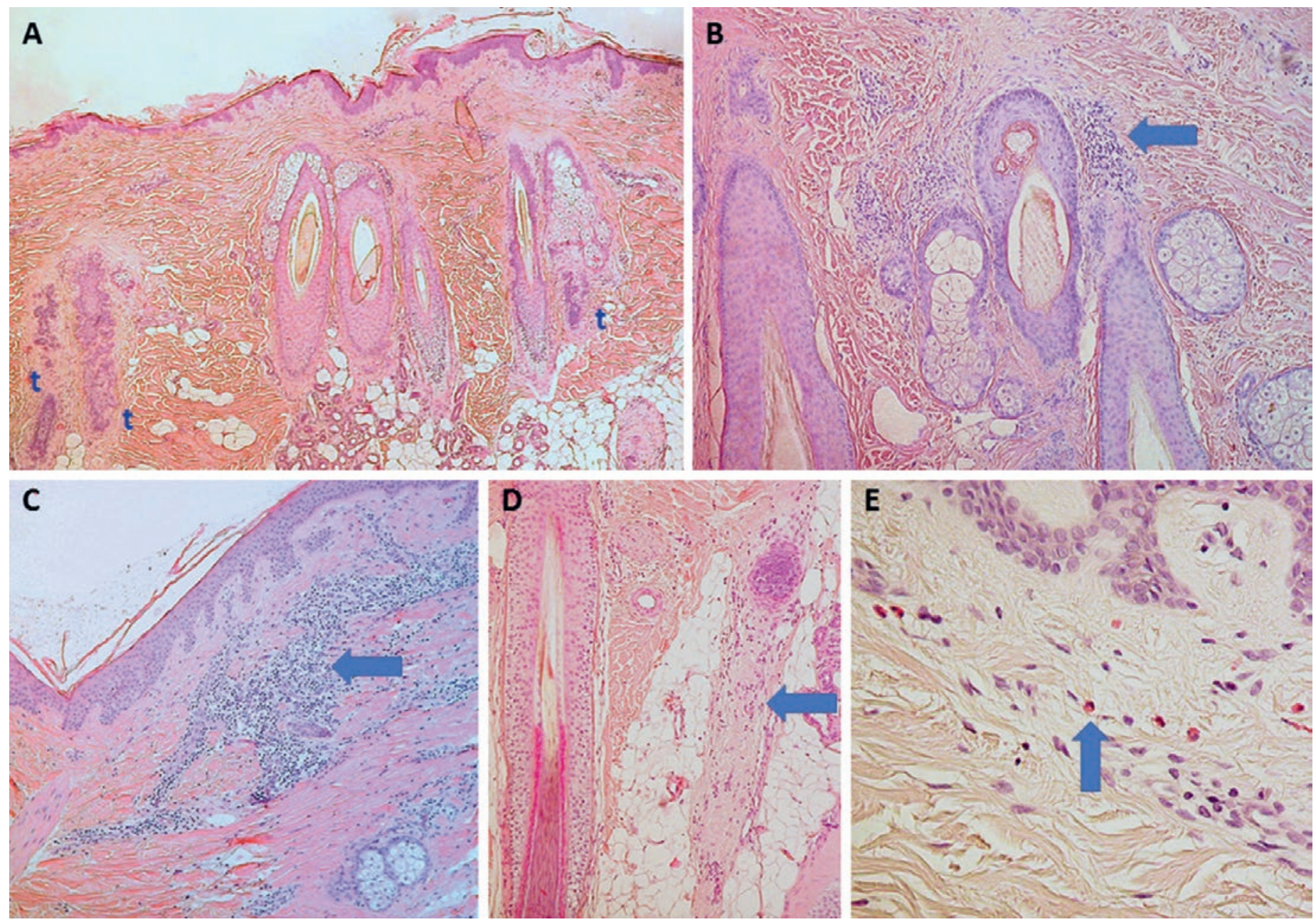

Abbildung 3: Alopecia areata incognita. Von der klassischen Alopecia areata («peribulbäres Bienenschwarm-Infiltrat») unterschiedliche Histologie (alle Abbildungen in Hämatoxylin-Eosin-Färbung). A: Vermehrte Telogenhaare (t) (Originalvergrösserung 10x). B: Hoch-perifollikuläres Infiltrat im Bereich der Talgdrüsen (Pfeil) statt tief-follikulär am Bulbus (20x). C: Superfiziell perivaskuläres Infiltrat (Pfeil) (20x). D: Infiltrat in den fibrösen Trakten der Telogenhaare (Pfeil) (20x). E: Eosinophile im Infiltrat (Pfeil) (40x). Meist liegen die Elemente unvollständig und nur diskret vor (grosse Biopsie notwendig).

\section{Vernarbende Alopezien}

Die AGA kann in seltenen Fällen vernarbend ablaufen, mit Fibrosierung der Follikel. In diesen Fällen ist ein Zurückwachsen der Haare unter Therapie nicht möglich, nur ein Stoppen der Progression. Extremformen der vernarbenden AGA stellen die fibrosierenden Alopezien dar, zuweilen als Typus alleine, zuweilen kombiniert auftretend. Sie betreffen meist peri- oder postmenopausale Frauen, können aber auch bei jungen Patientinnen und bei Männern beobachtet werden. Die frontale fibrosierende Alopezie (Morbus Kossard) entspricht dem männlichen Verteilungsmuster der AGA und lässt die frontale Haarlinie nach hinten weichen, bis hin zum Vertex ("Clown-Alopezie»). Typisch ist ein narbiges, glattes weissliches frontales Band mit - pathognomisch - auch haarloser Zone vor den Ohren; die Abgrenzung gegenüber der chronisch sonnengeschädigten (fleckigen und gefältelten) Stirn ist deutlich; die Haarfollikelöffnungen der verbleibenden Haare sind oft etwas hyperkeratotisch und diskret gerötet; nicht immer klagen die Patienten über moderaten Juckreiz. Häufig geht dem Haarausfall ein Verlust der Augenbrauen voraus. Das Pendant im weiblichen AGA-Muster betrifft das Zentrum des Kopfes am Vertex (Morbus Trüeb); vieles, was früher als Pseudopelade Brocq beschrieben wurde, entspricht diesem Alopezietyp. Auch ist zuweilen der hippokratische Kranz hinter den Ohren und am Occipitum betroffen. Histologisch findet sich ein Entzündungsmuster, welches weitgehend einem Lichen planopilaris entspricht: bei entsprechendem mikroskopischem Befund obliegt es dem Kliniker, die korrekte Schlussfolgerung zu ziehen. Aufgrund ihrer histologischen Charakteristika werden die fibrosierenden Alopezien zuweilen noch als Sonderform des Lichen planopilaris klassiert; die Behandlung als solche führt jedoch kaum jemals zum 
Erfolg, während eine energische Anti-AGA-Therapie mit einem potenten Inhibitor der 5a-Reduktase (Dutasterid [off-label]) in den meisten Fällen zum Sistieren der Progression führt [5]; ein Nachwachsen der Haare ist auch hier wegen der Vernarbung ausgeschlossen, weswegen die frühe Erkennung und Behandlung wichtig ist. Interessant ist, dass der frontale Typ dieses Haarausfalls erst seit zirka 20 Jahren beobachtet wird, aber mit rapide zunehmender Häufigkeit; wegen überzufälliger Assoziation mit Verwendung von Kosmetika und Sonnenschutzmitteln ist eine negative Wirkung von in diesen enthaltenen, hormonell wirksamen Chemikalien als endokrine Disruptoren nicht ausgeschlossen.

Von den fibrosierenden Alopezien abzugrenzen sind weitere vernarbende Alopezien, die im AGA-Gebiet vornehmlich bei Migranten und vereinzelt noch bei älteren Damen auftreten: Traktionsalopezie (an der Stirn als Alopecia marginata, am Vertex als ChignonAlopezie) und Druckalopezie durch zu enge Kopftücher (Abb. 4A-D). Anfänglich noch reversibel, erfolgt bei langanhaltender Schädigung die Vernarbung, weswegen die Therapie im Aufzeigen der Ursache liegt; leider besteht häufig Beratungsresistenz.
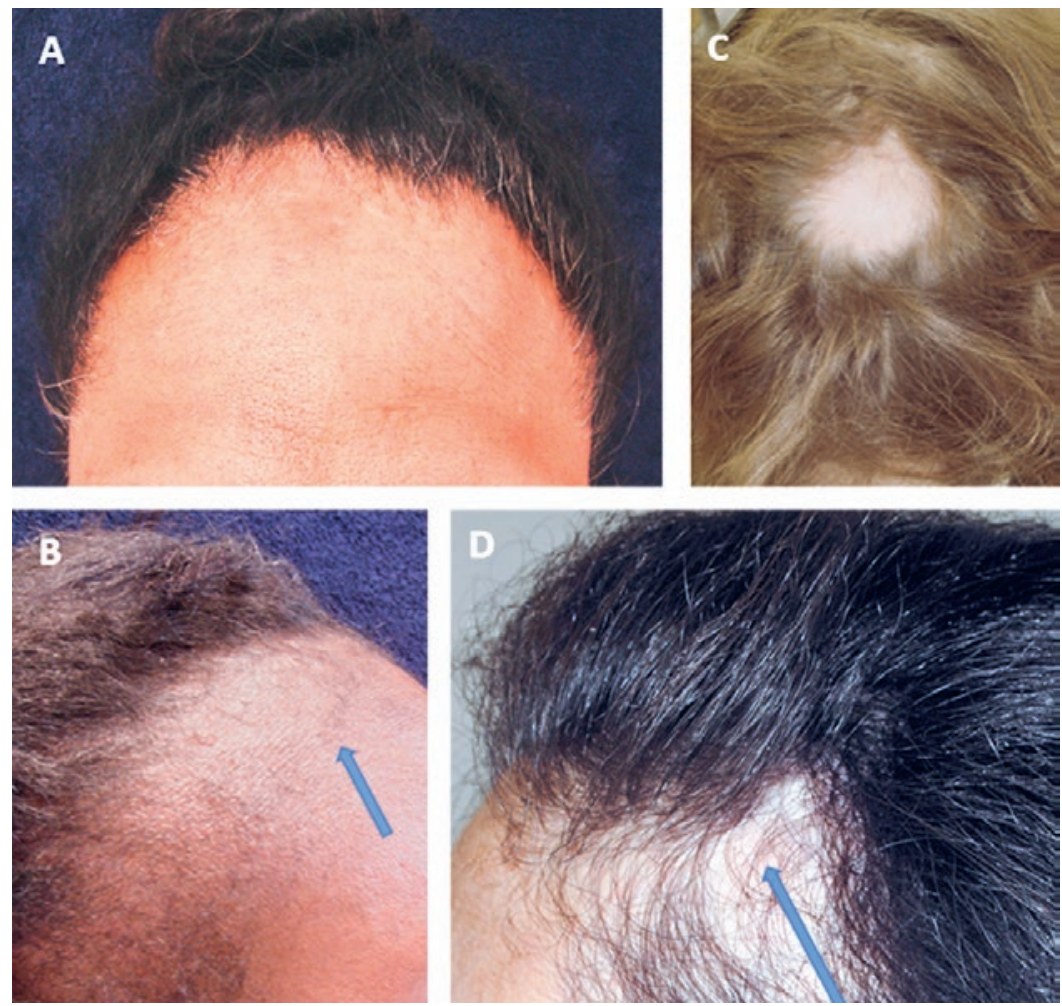

Abbildung 4: A: Traktionsalopezie bei Sikh (Haare unter dem Turban verstaut; kein Haarsaum, weil Druckalopezie durch Turban). B: Traktionsalopezie bei Afrikanerin (beachte den kleinen übrigbleibenden Saum von Haaren, die nicht gefasst werden können [Pfeil]: Alopecia marginata). C: Traktionsalopezie bei älterer Dame durch «Bürzi»/Dutt (ChignonAlopezie). D: Druckalopezie bei Muslima durch zu eng gefasstes Kopftuch (beachte leichte Lichenifikation [Pfeil]).

\section{Kongenitale trianguläre temporale Alopezie} und andere konstitutionelle Simulatoren

Die kongenitale trianguläre temporale Alopezie ist kaum jemals kongenital oder triangulär, sondern erscheint im Verlauf der Kindheit als haarlose, mit spärlichstem Flaum bedeckte, umschriebene rundliche Zone im fronto-temporalen Bereich. Wenn hinter der Haarlinie liegend, besteht die Verwechslungsgefahr mit einer Alopecia areata. Wenn sie aber beidseitig und am Rand des Haaransatzes auftritt, können vertiefte Geheimratsecken angenommen werden. Weil die Haarlosigkeit zuweilen erst im jungen Erwachsenenalter bemerkt wird, ist die gängige Erklärung später Aufmerksamkeit wenig plausibel; wahrscheinlicher handelt es sich um eine hamartielle In- oder Aktivierung eines für das Haarwachstum relevanten Gens, z.B. von hr (hairless). Behandlung der Wahl ist die Therapieabstinenz oder eine Haartransplantation.

Als normaler Haarverlust gilt tatsächlich nur, was in der Bilanz zwischen Ausfallen und Nachwachsen nicht zu einer über für das Alter Normale hinausgehenden Haarausdünnung führt.

Die unselige tradierte Lehre, dass ein Verlust von bis zu 100 Haaren pro Tag normal sei, hat sich auch im Laienunwissen festgesetzt. Tatsächlich ist aber nur normal, was in der Bilanz zwischen Ausfallen und Nachwachsen nicht zu einer über für das Alter Normale hinausgehenden Haarausdünnung führt. Es gibt Patientinnen (und Ärzte), die völlig auf diese magische Zahl fixiert sind und ihre Haare täglich zählen, obwohl deren gegebenenfalls auch objektiv starker Turnover nicht zur pathologischen Ausdünnung führt (sog. chronisches Telogeneffluvium); sie beklagen zwar keine Alopezie, aber fürchten sie; eine standardisierte Fotografie kann zur Beruhigung führen. Schwieriger sind Patientinnen mit schönem Haar, die selbst bei üblichem Turnover einen krankhaften Haarausfall beklagen (psychogenes Pseudoeffluvium); hier handelt es sich um Persönlichkeitsstörungen mit Dysmorphophobie, welche bis zum Suizid führen kann. Diese Patientinnen sind aber sehr selten und die Diagnose ist selbstkritisch und nur als Ausschlussdiagnose zu stellen. Bei konstitutioneller Hypotrichose wird die physiologische AGA schnell pathologisch wirkende Ausmasse annehmen. Bei der Myotonen Dystrophie Typ 1 Curschmann-Steinert und beim Trichorhinophalangeal-Syndrom ist eine AGA-analoge Haarauslichtung oft früh ausgeprägt, wobei neben Störungen im peripheren Androgenmetabolismus auch Störungen der Haardifferenzierung diskutiert werden. 
Korrespondenz:

Dr. med. Pierre de Viragh

Facharzt für Dermatologie,

spez. Dermatopathologie

Schanzeneggstrasse 1

CH-8002 Zürich

info.dermadoc[at]bluewin.ch

\section{Schlussfolgerungen}

Die erfolgreiche Therapie der AGA ist möglich. Falsch ist das Studentenwissen, dass zur AGA keine Differentialdiagnose besteht. Gerade das Nicht-Beachten der Simulatoren führt zum Therapieversagen, welches Pa-

\section{Das Wichtigste für die Praxis}

- Die androgenetische Alopezie (AGA) ist ein normaler Alterungsprozess. Bei für das Alter übermässiger Ausprägung ist bei Mann und Frau eine erfolgreicheTherapie oft möglich.

- Therapieversagen ist oft die Folge des Nicht-Beachtens von Simulatoren. Diese müssen bei auffälliger Anamnese (plötzlicher Beginn oder plötzliche Verschlechterung, Beschleunigung) oder ungewöhnlicher Klinik bedacht werden.

- Die wichtigsten die AGA verstärkenden oder imitierenden Ursachen sind bei der menstruierenden Frau der Eisenmangel, auch ohne Anämie, und generell die iatrogenen Störungen ihrer Hormonlage.

tientin und Arzt zugleich frustrieren. Bei umfassender Beurteilung mit gezielter Anamnese statt unstrukturiertem Gespräch, kleinem Labor und dann nachhaltiger Therapie eventueller Simulatoren, wird aus der «Strafsprechstunde» wieder eine erfolgreiche Haarsprechstunde.

Disclosure statement

Der Autor hat keine finanziellen oder persönlichen Verbindungen im Zusammenhang mit diesem Beitrag deklariert.

\section{Literatur}

1 von Wolff M, de Viragh PA, Stute P. Androgenisierungserscheinungen. Gynäkologie. 2011;3:10-15. Available from www.rosenfluh.ch/ media/gynaekologie/2011/03/androgenisierungserscheinungen.pdf

2 Levy LL, Emer JJ. Female pattern alopecia: current perspectives. Int J Womens Health. 2013;5:541-56. (open access full text article)

3 Kantor J, Kessler LJ, Brooks DG, Cotsarelis G. Decreased serum ferritin is associated with alopecia in women. J Invest Dermatol. 2003;121:985-8.

4 de Viragh PA. Ferropenic alopecia. In: Camacho F, Tosti A (eds.). Montagna Trichology - Diseases of the Pilosebaceaous Follicle. 2 vols. (3. ed.) Madrid: Aula Medica. 2017:735-42.

5 Vañó-Galván S, et al. Frontal fibrosing alopecia: a multicenter review of 355 patients. J Am Acad Dermatol. 2014;70:670-8. 